\title{
Genotyping and phylogenetic placement of Bacillus anthracis isolates from Finland, a country with rare anthrax cases
}

\author{
Taru Lienemann', Wolfgang Beyer ${ }^{2}$, Kirsti Pelkola' ${ }^{1}$, Heidi Rossow ${ }^{3}$, Alexandra Rehn ${ }^{4}$, Markus Antwerpen $^{4}$ \\ and Gregor Grass ${ }^{4^{*}}$
}

\begin{abstract}
Background: Anthrax, the zoonotic disease caused by the gram-positive bacterium Bacillus anthracis, is nowadays rare in northern parts of Europe including Finland and Scandinavia. Only two minor outbreaks of anthrax in 1988 and in 2004 and one sporadic infection in 2008 have been detected in animals in Finland since the 1970's. Here, we report on two Finnish $B$. anthracis strains that were isolated from spleen and liver of a diseased calf related to the outbreak in 1988 (strain HKI4363/88) and from a local scrotum and testicle infection of a bull in 2008 (strain BA2968). These infections occurred in two rural Finnish regions, i.e., Ostrobothnia in western Finland and Päijänne Tavastia in southern Finland, respectively.
\end{abstract}

Results: The isolates were genetically characterized by PCR-based methods such as multilocus variable number of tandem repeat analysis (MLVA) and whole genome-sequence analysis (WGS). Phylogenetic comparison of the two strains HKI4363/88 and BA2968 by chromosomal single nucleotide polymorphism (SNP) analysis grouped these organisms within their relatives of the minor canonical A-branch canSNP-group A.Br.003/004 (A.Br.V770) or canonical B-branch B.Br.001/002, respectively. Strain HKI4363/88 clustered relatively closely with other members of the A.Br.003/004 lineage from Europe, South Africa, and South America. In contrast, strain BA2968 clearly constituted a new sublineage within B.Br.001/002 with its closest relative being HYO01 from South Korea.

Conclusions: Our results suggest that Finland harbors both unique (autochthonous) and more widely distributed, common clades of B. anthracis. We suspect that members of the common clades such as strains HKI4363/88 have been introduced only recently by anthropogenic activities involving importation of contaminated animal products. On the other hand, autochthonous strains such as isolate BA2968 probably have an older history of their introduction into Finland as evidenced by a high number of single nucleotide variant sites in their genomes.

Keywords: Bacillus anthracis, Finland, Whole genome sequencing (WGS), Comparative genomics, Single nucleotide polymorphism (SNP), Multiple locus variable number of tandem repeat analysis (VNTR, MLVA)

\section{Background}

The zoonosis anthrax is often referred to as a neglected tropical disease [1]. This is because most animal and human cases occur in (sub)tropical countries of Sub-Sahara Africa and parts of Asia [2]. However, in the past the bacterium causing anthrax, Bacillus anthracis, had a much wider distribution. For instance, high latitude

\footnotetext{
* Correspondence: gregorgrass@bundeswehr.org

${ }^{4}$ Bundeswehr Institute of Microbiology, Munich, Germany

Full list of author information is available at the end of the article
}

countries such as Sweden, Finland, Canada or the area of the Russian Federation (i.e., the former Soviet Union) had also been affected by the disease, regularly. In Finland, altogether 283 anthrax cases in 150 different localities have been detected in several animal species since 1940's (Zoonoses in Finland in 2000-2010. online available: https://www.evira.fi/globalassets/elaimet/zoono osikeskus/zoonoosit/zoonosesinfinland_final_nettiversio. pdf. Accessed July 11, 2018). The majority of anthrax outbreaks involved bovines, but also other animal species such as horses, swine, sheep, dogs and fur animals 
like minks and foxes have been affected. Due to specific control measurements, improved feed hygiene and awareness of the epidemiology of anthrax, the disease is nowadays rare in Finland. However, B. anthracis still causes large outbreaks elsewhere at high latitudes. For instance, after the last outbreak in 1941 in a northern, boreal part of what is now the Yamal-Nenets autonomous district located within the Russian Federation, 2.657 infected animals were reported from six different outbreaks in the summer of 2016. From these animals and their products several human fatalities had resulted in the aftermath [2]. Besides, several outbreaks occurred among farm animals in southeastern Sweden in the same summer [2]. In Canada, anthrax is still enzootic in central parts of the country and free-roaming Wood Bisons are frequently affected during summer months [3].

While there is an increasing number of available genome sequences of $B$. anthracis from broad geographic origins, there are still only a few sequenced isolates from higher latitudes. From Norway, only two multilocus sequence typed bovine isolates originating from two outbreaks in 1987 and 1993, respectively [4], and a few genomes from bovine isolates partly lacking further meta-data have been reported $[5,6]$. Conversely, a prominent Norwegian B. anthracis genome is the one originating from imported contaminated heroin; the isolate causing the very first fatality of injectional anthrax in 2000 [7]. From Sweden stems one genome and very closely related isolates from a primary outbreak at a nature reserve in 2011. This outbreak was likely caused by the disturbance of a historical animal burial site and resulted in a secondary outbreak in 2013 [8]. Other strains originate from older collections as in the case of Denmark [9] or Finland (this study) because nowadays anthrax is a very rare disease in these countries, too.

In this study, we genotyped and whole genome-sequenced the only two available $B$. anthracis isolates from Finland. One strain (HKI4363/88) originated from an outbreak in Ostrobothnia, western Finland, in August 1988 [10]. Isolate BA2968, was isolated in Helsinki in September 2008 from an aspirate of an oedematous scrotum of a young bull (born in 2007) which was diagnosed with a testicle and scrotum inflammation.

The new genomic data from the Finnish isolates was used to compare these $B$. anthracis isolates with their close relatives and to assign the Finnish strains' placements in a phylogenetic context.

\section{Methods}

\section{Growth of B. anthracis and extraction of DNA}

Vegetative cells of $B$. anthracis from our strain collections were cultured on blood agar, subsequently inactivated and DNA was isolated as described previously [11] using DNeasy Blood and Tissue kit (Qiagen, Germany) as described for Gram-positive bacteria with the following minor modifications. After cell wall lysis with lysozyme $(20 \mathrm{mg} / \mathrm{ml}), 4 \mu \mathrm{l}$ of RNase A $(100 \mathrm{mg} / \mathrm{ml})$ was added and the suspension was incubated for $2 \mathrm{~min}$ at room temperature. DNA was eluted twice $(50 \mu \mathrm{l}$ and $50 \mu \mathrm{l})$ using sterile nuclease-free water. Handling of live $B$. anthracis occurred in a biosafety level 3 laboratory and the isolated DNA was sterile-filtered $(0.22 \mu \mathrm{m}$ filter pore, Merck Millipore, Germany) prior to being taken from biosafety level 3 laboratory. DNA concentrations were quantified using the Qubit dsDNA HS Assay Kit (Thermo Fisher Scientific, USA) according to the supplier's protocol. DNA preparations were stored at $-20{ }^{\circ} \mathrm{C}$ until further use.

\section{Diagnostic real-time PCR for chromosomal and plasmid markers of $B$. anthracis}

Three specific genetic markers including chromosomal marker dhp61 (BA_5345) as well as plasmid markers pagA (pXO1) and capC (pXO2) were used to identify $B$. anthracis by real-time PCR as described in [12, 13]. Real-time PCR assays were conducted using a LightCycler 480 II (Roche, Germany) and data analysis was performed with the associated instrument software.

\section{Analysis of canonical single nucleotide polymorphisms (canSNPs)}

Isolates were grouped into the canonical SNP (canSNP) typing scheme that allows attribution into genetic groups within the accepted global population structure of B. anthracis [14]. For this, Mismatch Amplification Mutation Assays (Melt-MAMA) [15] for 12 canSNP groups of $B$. anthracis-isolates were performed (primer sequences in Additional file 1) on a LightCycler 480 II instrument (Roche, Germany) as described in [13] and data analysis was performed with the associated instrument software.

\section{Multi locus variable number of tandem repeats analysis using 31 markers (MLVA-31)}

The MLVA was performed essentially as described in [16]. Briefly, amplification of the fragments of 31 marker-loci was performed in 7 multiplex-PCRs (the origins of the best matches to the Finnish $B$. anthracis isolates are listed in Additional file 2). The fragment-mixtures were analyzed on a Genetic Analyzer (ABI 3130, Applied Biosystems, Germany) using either MegaBACE TMET (GE Healthcare, Germany), Genescan 1200 LIZ (Applied Biosystems, USA) or MapMarkerH 1000 (BioVentures, USA) as size standards. The data were analyzed with GeneMapper TM software (Applied Biosystems, USA). The raw data of fragment lengths were normalized by codes, reflecting the actual copy numbers of the repeat sequences where possible. 
Whole genome sequencing and assembly

The Nextera ${ }^{\circledR}$ XT DNA Library Preparation kit (Illumina, USA) was used for library preparation with DNA inputs of 1 to 3 ng per library. Libraries were sequenced on a MiSeq instrument (Illumina, USA) using MiSeq Reagent Kit v3 (600-bp) chemistry (Illumina, USA). High-quality paired-end reads $(\mathrm{Q} \geq 30)$ were assembled de novo into draft genomes using an in-house script based on the SPAdes assembler (version 3.11.1) [17]. The automated genome refining tool Pilon (version 1.22) [18] was used to further improve these draft genomes by correcting SNPs or closing small gaps. All processed scaffolds were manually checked for contaminant reads and uploaded to the NCBI Bioproject database (The data-set supporting the conclusions of this article is available in the Bioproject PRJNA309927, accession numbers: Additional file 3). Annotation was automatically performed by the NCBI Prokaryotic Genome Annotation Pipeline [19].

Analysis of whole genome sequencing data - SNP calling The Parsnp tool from Harvest Suite was used for rapid core chromosome multiple-alignment [20]. Input data for this were representative $B$. anthracis genomes from public databases (Additional file 3) and newly sequenced strains that were aligned against the 'Ames ancestor' reference chromosome (NC_007530) using Parsnp (parameters -c -e -u -C 1000). Called SNPs were extracted into a VCF file using HarvestTools (version 1.0) from the same software suite. To enhance overall data quality, SNP positions with a distance of less than $10 \mathrm{bp}$ as well as positions harboring an undefined nucleotide ("N") were removed. The " $\mathrm{R}$ " analysis package phangorn (version 2.4.0) was used to determine homoplasious SNPs (parameter "CI(tree, data, cost = NULL, sitewise = TRUE )") and to remove them [21]. This edited file served again as an input file in the HarvestTools to compile a FASTA file comprising a multiple-sequence alignment of the concatenated SNPs. Next, the evolutionary history was inferred from this data by using the Maximum Likelihood method according to the Tamura-Nei model [22]. A phylogenetic maximum likelihood tree was computed in Mega7 [23] and a minimum spanning tree was computed in BioNumerics 6.6 (Applied Maths, Belgium) from the VCF SNP-file (in binary format) as input and manually edited for style.

\section{Results}

\section{Genotyping of two B. anthracis isolates from Finland}

Isolates HKI4363/88 and BA2968 were classified within the three major branches $\mathrm{A}, \mathrm{B}$ and $\mathrm{C}$ and assigned to canonical SNP-groups of B. anthracis [14]. HKI4363/88 belonged to the A-branch (A.Br.) A.Br.003/004 defined by an ancestral SNP state for SNP A.B.03 and a derived state for A.Br.004. According to a recently amended nomenclature, this major branch is now called A.Br.V770 defined by the SNP-states of A.Br.003 and A.Br.054 [24]. Isolate BA2968 grouped within the B-branch, B.Br.001/002, typically comprising European and African strains [14].

In order to identify possible close relatives of these Finnish strains, we performed MLVA-31 typing and compared the strains' profiles with that of our in-house MLVA-database. Additional file 4 summarizes the results of MLVA-31 for strains HKI4363/88, BA2968 and their closest matches. Next, we used this data to visualize the MLVA-similarities between Finnish strains and relatives from other geographical origins (Fig. 1). Finnish isolate HKI4363/88 clustered closely (6 markers difference) with a strain isolated around Cape Town (Rondebosch) in 1999. Near relatives of this strain HKI4363/88 (five markers difference) had also been found in a soil sample of a derelict tannery (Neumünster, Germany) with a > 100 year history, namely isolate A155. Isolate A172 originated from an outbreak in bovines (four markers difference) in France in the year 2001. Interestingly, there is also a more distant relation (seven markers difference) to the B. anthracis type strain V770 NP/1 (ATCC 14185) used for human vaccine production in the USA for a long time. Another relative of this group (A142) was found at the same tannery site as strain A155. From the MLVA-31 results presented in Fig. 1, we selected seven strains from our strain collections most similar in their VNTR-profile to Finnish isolates HKI4363/88 or BA2968, respectively, for whole genome sequencing (Additional file 3).

Chromosomal SNP analysis suggests that Finnish B. anthracis strains comprise both an autochthonous lineage and introduction via more recently importation events Genome sequencing of the two Finnish isolates HKI4363/88 and BA2968 as well as strains A16, A24, A46, A142, A155 and SA047 yielded an average number of 612,000 reads $(588,000-660,000)$ per isolate, resulting in an average sequencing depth of $>52$-fold. De novo assembly produced between 22 and 41 scaffolds (>500 bp) per genome. Each isolate covered at least $99.95 \%$ of the reference chromosome of $B$. anthracis str. 'Ames ancestor' (NC_007530).

From this chromosomal dataset of the in-house sequenced strains and representatives from public databases (Additional file 3) 3211 non-homoplasious SNPs were called of which 1548 were parsimony informative sites (Additional file 5). The concatenated chromosome-wide SNPs were used to infer the phylogenetic relationships of the analyzed strains with focus on the A.Br.003/004 and all three B-lineages (Fig. 2). Chromosomes from strains of neighboring canSNP groups A.Br.Ames, A.Br.001/002 Sterne and A.Br.003/004 V770 were included for reference. 


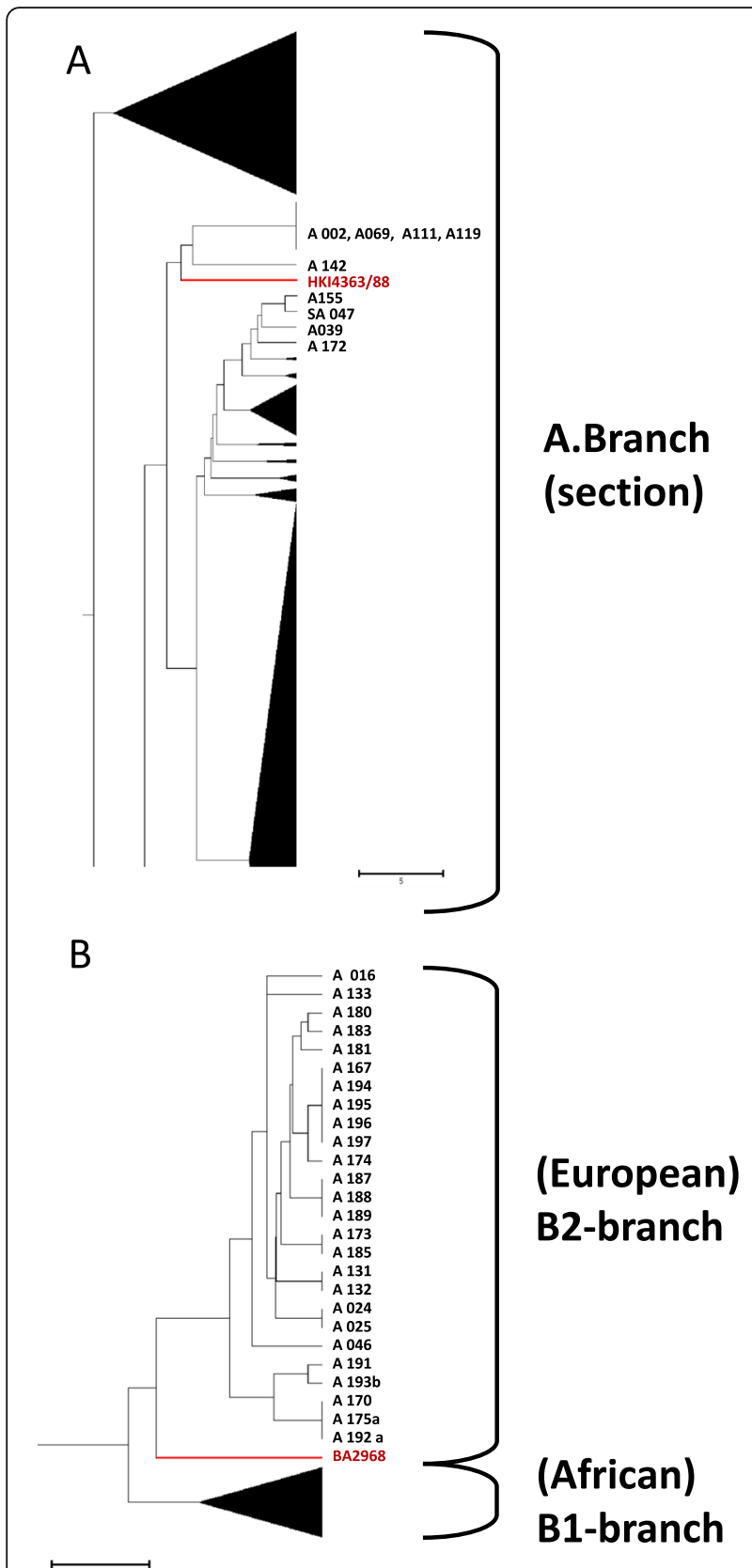

Fig. 1 Position of strains HKI4363/88 and BA2968 in UPGMA cluster analysis based on 31 B. anthracis MLVA markers. Positions of strains HKI4363/88 (a) and BA2968 (b) (indicated in red) among their closest MLVA-relatives within a data-set of 976 B. anthracis isolates are shown

Within the phylogeny, strain HKI4363/88 clustered relatively closely with other members of the A.Br.003/004 lineage including strains from Germany, South Africa, Argentina, Bolivia, and Chile and more loosely with isolates from the United States of America. Notably, the other Finnish strain BA2968, positioned within canSNP group B.Br.001/002 as the sole member of a sister clade to the one leading to strains from South Korea, South Africa,
Zimbabwe and Sweden as well as including the branch leading to canSNP group CNEVA (Fig. 2).

When focusing on SNP-differences between isolated strains it became obvious that strain BA2968 was unique, clearly constituting a new sublineage within B.Br.001/002. This isolate was separated by 215 SNPs to its closest relative, HYO01, from South Korea and 256 SNPs to canSNP-group B.Br.CNEVA isolate BF-1 from Germany (Additional file 6). Conversely, strain HKI4363/ 88 was separated by only 52 SNPs distance to strain A1096 from South Africa and less than 100 SNPs to a cluster of isolates from Germany, Bolivia, Argentina, Chile and the United States of America. Of note, the German strains from this group were isolated from the site of a derelict tannery and thus have been likely imported via contaminated animal products (W. Beyer, unpublished).

\section{Discussion}

The two isolates from Finland offer a rare glimpse into the past genomic diversity of $B$. anthracis in European high northern latitudes. During the small outbreak in western Finland in 1988, from which strain HKI4363/88 was isolated, two out of 14 bovines, one heifer and one-year-old calf were infected with $B$. anthracis with typical sudden anthrax symptoms of tremble, fever, paralysis and bleeding. Prior death the calf was treated with penicillin and was bleeding from the anus. Both animals died a couple of hours after the onset of the symptoms. Anthrax was suspected by a municipal veterinarian and B. anthracis was isolated from liver and spleen and confirmed by Giemsa staining, animal experiment, and later by PCR at EVIRA (Finnish Food Safety Authority). At the same farm where the two diseased animals were kept in 1988, two earlier cases of anthrax had been documented in 1986. Furthermore, within the immediate surrounding of the farm, anthrax had been documented 30 years earlier [10].

Strain BA2968 was sampled from a bull examined at Saari clinic at the production animal hospital of the University of Helsinki, where bacteria belonging to the genus Bacillus were detected in the scrotum aspirate culture. The Bacillus isolate was examined further at EVIRA by PCR and identified as B. anthracis. The bull was culled immediately after the diagnosis. Blood samples of all the cows with fever from the same farm were examined for $B$. anthracis with negative results. At the same farm where the bull was kept, two cows had died of anthrax 4 years earlier. In addition, diseased bovine animals had been buried in the area several decades ago and also a factory rendering animal byproducts had been run many years ago upstream of a ditch flowing into the farm area. This factory had also processed imported material of animal origin and possibly contaminated the surroundings. Thus, the $B$. anthracis strain causing this 


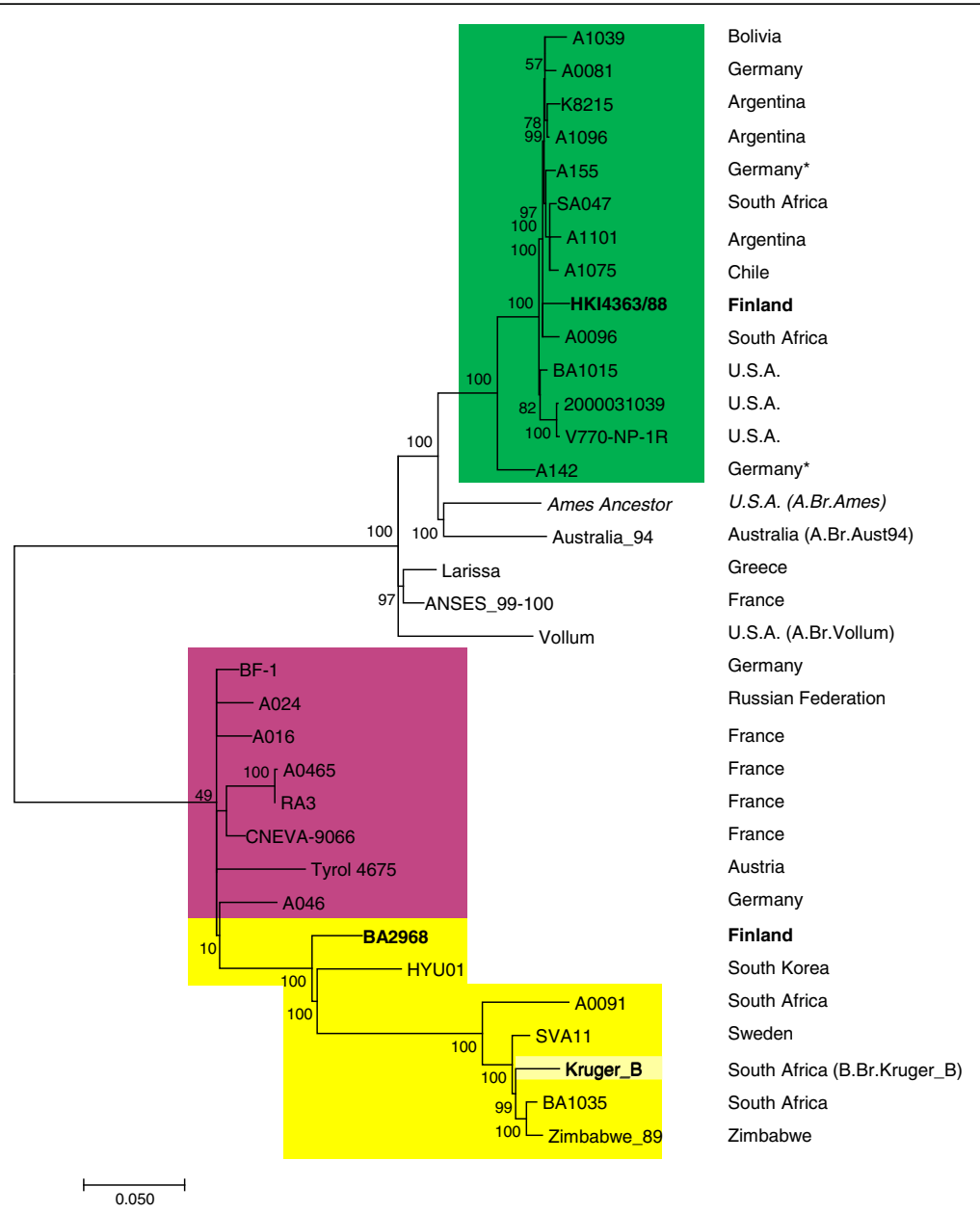

Fig. 2 Unrooted phylogenetic tree of representative B. anthracis strains derived from chromosomal SNPs. The evolutionary history of B. anthracis strains was inferred from 1,548 non-homoplasious chromosomal SNPs by using the Maximum Likelihood method based on the Tamura-Nei model [22]. The tree is drawn to scale, with branch lengths measured in the number of substitutions per site. Colors indicate canSNP groups A.Br.003/004 (A.Br.V770, green), B.Br.CNEVA (purple) or B.Br.001/002 (yellow), respectively. Bootstrap values (based on 1000 replications) are given as percentages at branch nodes. The right column indicates the country of origin and canSNP group information of representatives (in brackets)

"cutaneous" anthrax was probably introduced into the scrotum tissue through a skin wound or abrasion by contaminated soil material after a very rainy summer and autumn. Because of the close vicinity of anthrax outbreaks to the factory processing also imported animal byproducts, it is possible that isolate BA2968 does not represent an autochthonous $B$. anthracis strain but one that has been anthropologically introduced.

In northern latitude countries such as Finland and Sweden, anthrax control programs of the past have resulted in an increasingly rare occurrence of the zoonosis today. In Sweden, a ban on the import of bone meal for animal feed in 1957 led to a steep decline in new outbreaks within a very few years [25]. In the last half century, only outbreaks in 1981, 2008, the most likely linked outbreaks of 2011/13 and a last one in 2016 were documented $[8,25]$. Thus, characterization of isolates from such rare events can provide unique opportunities to record remnants of the genetic diversity of the all but extinct pathogen in this country. Possibly, in Sweden and Norway as well, there will be a situation similar to that in Finland with B. anthracis genotypes recovered that may be either autochthonous or originate from importation of contaminated animal products such as wool, hides or bone meal.

Taking the phylogeography of $B$. anthracis isolate BA2968 into account, our data indicates a common origin of this and other strains belonging to the so-called (European) B2-Branch [26] isolated in central Europe. The European B-Branch, as originally specified by MLVA, is a wide-spread group of $B$. anthracis strains which are most probably of African origin [27].

\section{Conclusions}

There is some information on the genomic diversity on $B$. anthracis from higher northern latitudes. Nevertheless, it 
would be worthwhile to mine the genomes of further isolates present in older collections of this pathogen. Such efforts would advance our capabilities in differentiating natural outbreaks in geographic locations where anthrax is very uncommon from deliberate releases of the pathogen. With that kind of information it will then be possible to conduct bioforensically sound outbreak related trace-back analysis and attribution.

\section{Additional files}

Additional file 1: Melt-MAMA canSNP primer sequences. (XLSX $11 \mathrm{~kb}$ )

Additional file 2: Origin of near relatives of strain HKI4363/88 and strain BK2968. (XLSX 9 kb)

Additional file 3: Genome sequences accession numbers of Finnish and additional B. anthracis strains from publically available databases. (XLSX $11 \mathrm{~kb}$ )

Additional file 4: MLVA-31 matrix of strains HKI4363/88 and BA2968 and their closest matches. (XLSX $22 \mathrm{~kb}$ )

Additional file 5: Chromosome-wide binary SNP matrix of analyzed genomes. (XLSX 530 kb)

Additional file 6: Position and SNP-distances of Finnish B. anthracis strains within their relatives in a Minimum Spanning Tree based on chromosomal SNPS. A Minimum Spanning Tree was inferred from 1,548 non-homoplasious chromosomal SNPs using the same dataset as in Fig. 2. Numbers next to branch lines indicate SNPs separating nodes or strains. The coloring is the same as in Fig. 2. (PDF $153 \mathrm{~kb}$ )

\section{Abbreviations}

bp: Base pairs; canSNP: Canonical Single Nucleotide Polymorphism; CNEVA: Centre National d'Etudes Veterinaires et Alimentaires; DDBJ/ENA: DNA Data Bank of Japan/ European Nucleotide Archive; DNA: Deoxyribonucleic acid; EVIRA: Finnish Food Safety Authority; FASTA: FAST All; MAMA: Mismatch amplification mutation assay; MLVA: Multiple locus variable number of tandem repeat analysis: NCBI: National Center for Biotechnology Information; PCR: Polymerase chain reaction; RNAse: Ribonuclease; SNP: Single nucleotide polymorphism; USA: United States of America; VCF: Variant call format; VNTR: Variable number of tandem repeat; WGS: Whole genome sequencing

\section{Acknowledgements}

Miriam Koene (Central Veterinary Institute of Wageningen University\&Research, Wageningen, The Netherland) is acknowledged for the generous gift of Dutch strains and Efi Petinaki (Department of Microbiology, Medical School, University of Thessaly, Thessaly, Greece) for providing strain Larissa. Thanks are due to Linda Dobrzykowski and Josua Zinner for skillful technical assistance.

\section{Funding}

The research described herein is part of the Medical Biological Defense Research Program of the Bundeswehr Central Medical Service. Opinions, interpretations, conclusions, and recommendations are those of the authors and are not necessarily endorsed by any governmental agency, department or other institutions. The funders had no role in study design, data collection and interpretation, writing the manuscript and the decision to submit the work for publication.

\section{Availability of data and materials}

The whole genome shotgun datasets generated in this study have been deposited at DDBJ/ENA/GenBank under the accessions QAEG00000000QAEN00000000. The versions described in this paper are versions QAEG01000000QAEN01000000.

\section{Authors' contributions}

G.G and H.R designed the study. T.L. isolated the B. anthracis DNAs of Finnish strains, K.P provided outbreak-related metadata and collected epidemiological information of anthrax in Finland. W.B., A.R. performed the experimental work and A.R. primary analysis. W.B. conducted MLVA-analysis, M.A., A.R. analyzed primary and publically available whole genome sequencing data. M.A., A.R. and G.G. performed bioinformatics data analysis. W.B. and M.A. made substantial contributions to guide the research and offered critical discussion of experimental results. G.G. M.A. and W.B. prepared the first draft of the manuscript. T.L., W.B., K.P. H.R., A.R., M.A. and G.G. reviewed and revised the manuscript; all authors read and approved the final manuscript.

Ethics approval and consent to participate

Not applicable

\section{Consent for publication}

Not applicable

\section{Competing interests}

The authors declare that they have no competing interests.

\section{Publisher's Note}

Springer Nature remains neutral with regard to jurisdictional claims in published maps and institutional affiliations.

\section{Author details}

${ }^{1}$ Finnish Food Safety Authority (Evira), Veterinary Bacteriology and Pathology Research Unit, Helsinki, Finland. ' $U$ niversity of Hohenheim, Stuttgart, Germany. ${ }^{3}$ Finnish Food Safety Authority (Evira), Risk Assessment Research

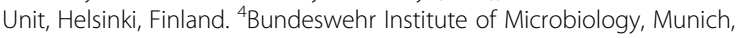
Germany.

Received: 18 April 2018 Accepted: 27 August 2018

Published online: 03 September 2018

References

1. Neglected zoonotic diseases. http://www.who.int/neglected_diseases/ zoonoses/infections more/en/. Accessed 11 July 2018.

2. Shadomy S, El Idrissi A, Raizman E, Bruni M, Palamara E, Pittiglio C, Lubroth J. Anthrax outbreaks: a warning for heightened awareness. In: Division APaH, editor. EMPRES Watch. Rome: Food and Agricultur Organization of the United nations (FAO); 2016. p. 8.

3. New D, Elkin B, Armstrong T, Epp T. Anthrax in the Mackenzie wood bison (Bison bison athabascae) population: 2012 anthrax outbreak and historical exposure in nonoutbreak years. J Wildl Dis. 2017;53(4):769-80.

4. Helgason E, Tourasse NJ, Meisal R, Caugant DA, Kolsto AB. Multilocus sequence typing scheme for bacteria of the Bacillus cereus group. Appl Environ Microbiol. 2004;70(1):191-201.

5. Vergnaud G, Girault G, Thierry S, Pourcel C, Madani N, Blouin Y. Comparison of French and worldwide Bacillus anthracis strains favors a recent, postColumbian origin of the predominant north-American clade. PLoS One. 2016;11(2):e0146216.

6. Johnson SL, Daligault HE, Davenport KW, Jaissle J, Frey KG, Ladner JT, Broomall SM, Bishop-Lilly KA, Bruce DC, Gibbons HS, et al. Complete genome sequences for 35 biothreat assay-relevant bacillus species. Genome Announc. 2015;3(2):e00151-15.

7. Keim P, Grunow R, Vipond R, Grass G, Hoffmaster A, Birdsell DN, Klee SR, Pullan S, Antwerpen M, Bayer BN, et al. Whole genome analysis of injectional anthrax identifies two disease clusters spanning more than 13 years. EBioMedicine. 2015;2(11):1613-8.

8. Ågren J, Finn M, Bengtsson B, Segerman B. Microevolution during an anthrax outbreak leading to clonal heterogeneity and penicillin resistance. PLoS One 2014;9(2):e89112

9. Derzelle S, Girault G, Kokotovic B, Angen O. Whole genome-sequencing and phylogenetic analysis of a historical collection of Bacillus anthracis strains from Danish cattle. PLoS One. 2015;10(8):e0134699.

10. Virtala A-MK, Tirkkonen TE, Pelkonen S. Translated title "an outbreak of anthrax". Language, Finnish. Journal, Eläinlääkäri: Suomen eläinlääkärilehti. 1988:94:606-10.

11. Braun P, Grass G, Aceti A, Serrecchia L, Affuso A, Marino L, Grimaldi S, Pagano S, Hanczaruk M, Georgi E, et al. Microevolution of anthrax from a young ancestor (M.A.Y.A.) suggests a soil-borne life cycle of Bacillus anthracis. PLoS One. 2015;10(8):e0135346.

12. Antwerpen MH, Zimmermann $P$, Bewley $K$, Frangoulidis D, Meyer $H$. Real-time PCR system targeting a chromosomal marker specific for Bacillus anthracis. Mo Cell Probes. 2008;22(5-6):313-5. 
13. Rume Fl, Ahsan CR, Biswas PK, Yasmin M, Braun P, Walter MC, Antwerpen M, Grass G, Hanczaruk M. Unexpected genomic relationships between Bacillus anthracis strains from Bangladesh and Central Europe. Infect Genet Evol. 2016:45:66-74.

14. Van Ert MN, Easterday WR, Huynh LY, Okinaka RT, Hugh-Jones ME, Ravel J, Zanecki SR, Pearson T, Simonson TS, U'Ren JM, et al. Global genetic population structure of Bacillus anthracis. PLoS One. 2007;2(5):e461.

15. Birdsell DN, Pearson T, Price EP, Hornstra HM, Nera RD, Stone N, Gruendike J, Kaufman EL, Pettus AH, Hurbon AN, et al. Melt analysis of mismatch amplification mutation assays (melt-MAMA): a functional study of a cost-effective SNP genotyping assay in bacterial models. PLoS One. 2012;7(3):e32866.

16. Beyer W, Bellan S, Eberle G, Ganz HH, Getz WM, Haumacher R, Hilss KA Kilian W, Lazak J, Turner WC, et al. Distribution and molecular evolution of Bacillus anthracis genotypes in Namibia. PLoS Negl Trop Dis. 2012; 6(3):e1534.

17. Bankevich A, Nurk S, Antipov D, Gurevich AA, Dvorkin M, Kulikov AS, Lesin VM, Nikolenko SI, Pham S, Prjibelski AD, et al. SPAdes: a new genome assembly algorithm and its applications to single-cell sequencing. J Comput Biol. 2012; 19(5):455-77.

18. Walker BJ, Abeel T, Shea T, Priest M, Abouelliel A, Sakthikumar S, Cuomo CA, Zeng Q, Wortman J, Young SK, et al. Pilon: an integrated tool for comprehensive microbial variant detection and genome assembly improvement. PLoS One. 2014:9(11):e112963.

19. Angiuoli SV, Gussman A, Klimke W, Cochrane G, Field D, Garrity G, Kodira CD, Kyrpides N, Madupu R, Markowitz V, et al. Toward an online repository of standard operating procedures (SOPs) for (meta)genomic annotation. OMICS. 2008;12(2):137-41.

20. Treangen TJ, Ondov BD, Koren S, Phillippy AM. The harvest suite for rapid core-genome alignment and visualization of thousands of intraspecific microbial genomes. Genome Biol. 2014;15(11):524.

21. Schliep KP. Phangorn: phylogenetic analysis in R. Bioinformatics. 2011;27(4): 592-3.

22. Tamura K, Nei M. Estimation of the number of nucleotide substitutions in the control region of mitochondrial DNA in humans and chimpanzees. Mol Biol Evol. 1993;10(3):512-26.

23. Kumar S, Stecher G, Tamura K. MEGA7: molecular evolutionary genetics analysis version 7.0 for bigger datasets. Mol Biol Evol. 2016;33(7):1870-4.

24. Sahl JW, Pearson T, Okinaka R, Schupp JM, Gillece JD, Heaton H, Birdsell D, Hepp C, Fofanov V, Noseda R, et al. A Bacillus anthracis genome sequence from the Sverdlovsk 1979 autopsy specimens. MBio. 2016;7(5):e00151-16.

25. Elvander M, Persson B, Sternberg Lewerin S. Historical cases of anthrax in Sweden 1916-1961. Transbound Emerg Dis. 2017;64(3):892-8.

26. Keim P, Price LB, Klevytska AM, Smith KL, Schupp JM, Okinaka R, Jackson PJ, Hugh-Jones ME. Multiple-locus variable-number tandem repeat analysis reveals genetic relationships within Bacillus anthracis. J Bacteriol. 2000; 182(10):2928-36.

27. Smith KL, DeVos V, Bryden H, Price LB, Hugh-Jones ME, Keim P. Bacillus anthracis diversity in Kruger National Park. J Clin Microbiol. 2000;38(10): $3780-4$.

Ready to submit your research? Choose BMC and benefit from:

- fast, convenient online submission

- thorough peer review by experienced researchers in your field

- rapid publication on acceptance

- support for research data, including large and complex data types

- gold Open Access which fosters wider collaboration and increased citations

- maximum visibility for your research: over $100 \mathrm{M}$ website views per year

At BMC, research is always in progress.

Learn more biomedcentral.com/submissions 